Recepción: 20 / 12 / 2017

Aceptación: 15 / 02 / 2018

Publicación: 8 / 05 / 2018
Ciencias Económicas

Artículo de Revisión

\title{
Análisis de los índices de GINI de Ecuador año 2017
}

Analysis of the GINI indices of Ecuador year 2017

Análise dos índices GINI do Equador no ano de 2017

Denis D. Navarro-Sangurima ${ }^{\text {I }}$

denis.navarros@ug.edu.ec

Mercy V. Agila-Maldonado II

mercy.agilam@ug.edu.ec

Ingrid I. Toala-Rocuano ${ }^{\text {III }}$

ingrid.toalar@ug.edu.ec

Correspondencia: denis.navarros@ug.edu.ec

\footnotetext{
${ }^{\mathrm{I}}$ Docente, Universidad de Guayaquil, Guayaquil, Ecuador.

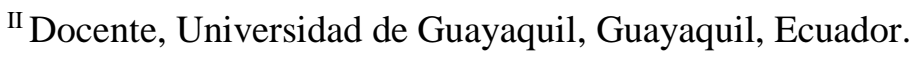

III Docente, Universidad de Guayaquil, Guayaquil, Ecuador.
} 


\section{Resumen}

El Coeficiente de Gini constituye un indicador el cual permite medir el grado de desigualdad de una variable en una distribución. El índice comprende valores desde cero (perfecta igualdad) hasta uno (perfecta desigualdad). A partir de aquello, dentro del presente manuscrito se describen aspectos generales donde se destaca apartados tales como Coeficiente de Gini: origen, evolución e institucionalización, historia y rasgos del coeficiente de Gini, parámetros de medición del coeficiente de Gini, metodología para el cálculo del coeficiente de Gini y los índices de Gini de Ecuador durante el año 2017, cada uno de estos apartados cuenta con el sustento bibliográfico que ayuda a una mejorar comprensión del lector, es decir, todo este enfoque desde una revisión bibliográfica.

Palabras Claves: Coeficiente de Gini, Curva de Lorenz, desigualdad, ingreso, riqueza 


\section{Abstract}

The Gini Coefficient is an indicator which allows to measure the degree of inequality of a variable in a distribution. The index includes values from zero (perfect equality) to one (perfect inequality). From that, within the present manuscript, general aspects are described, highlighting such sections as the Gini coefficient: origin, evolution and institutionalization, history and features of the Gini coefficient, measurement parameters of the Gini coefficient and methodology for calculating the Gini coefficient. Gini coefficient, each of these sections has the bibliographic support that helps a better understanding for its later application, that is, all this approach from a bibliographic review.

Key words: Gini coefficient, Lorenz curve, inequality, income, wealth 


\section{Introducción.}

El coeficiente de Gini es una medida que resume la manera en la que se distribuye el ingreso per cápita entre los habitantes de la población, mide el grado de desigualdad en la distribución del ingreso. Gini es un valor que se encuentra entre uno (ausencia de igualdad) y cero (igualdad perfecta). Resulta importante recalcar que, si bien el Coeficiente de Gini mide el grado de desigualdad de los ingresos, no constituye una medida directa para establecer el bienestar o las condiciones de vida de una determinada sociedad (INEC, 2017).

Desde el punto de vista estadístico, se comprende por "concentración" la forma como se reparte el total de una variable entre un conjunto de unidades agregadas o individuales. Es decir, se define en términos de la distribución de un monto total entre las n unidades. Por otro lado, la estadística ha desarrollado una serie de medidas que ayudan evaluar el grado de desigualdad de una distribución de frecuencias; entre ellas se destaca el coeficiente de concentración de Gini (Cortés \& Rubalcava, 2016).

Por otro lado, la naturaleza, causa e importancia de la desigualdad se encuentran abiertas a la discusión. Pero sin duda alguna, cada vez este término es más entendido como un problema, no únicamente ético, donde la distribución equitativa es entendida como un concepto de justicia social y solidaridad sino también en términos de eficiencia.

Bajo este contexto, posterior a la revisión bibliográfica se detalla el índice de Gini de Ecuador durante el año 2017, encontrando que en relación al año pasado este indicador sufrió cierta variación que en términos estadísticos no es muy significativo, sin embargo, se observa una disminución en la desigualdad que se registra en el país. 


\section{Desarrollo.}

El coeficiente de Gini representa una medida que permite determinar la concentración del ingreso en una sociedad, es decir que parte de la población agrupa determinada cantidad de recursos, Por lo que, el coeficiente de Gini ayuda a un Estado a tomar medidas en relación a políticas públicas que ayude a fiscalizar la distribución correcta de riquezas. Es decir, representa una medida de la desigualdad, es el ratio del área entre la línea de perfecta igualdad y la curva de Lorenz L(p) y el área debajo de la línea de perfecta igualdad, puede tomar valores entre 0 y 1 (Nabernegg, 2015), en el cual a medida que se acerca a cero se puede decir que hay una eficiente distribución del ingreso, y entre más se acerca a uno, se considera que una persona retiene todos los recursos en un territorio y periodo determinado. Sin embargo, el coeficiente de Gini es un indicador estadístico que considera únicamente dos variables, población e ingreso (Fuenzalida, Buzai, Morero \& García, 2015).

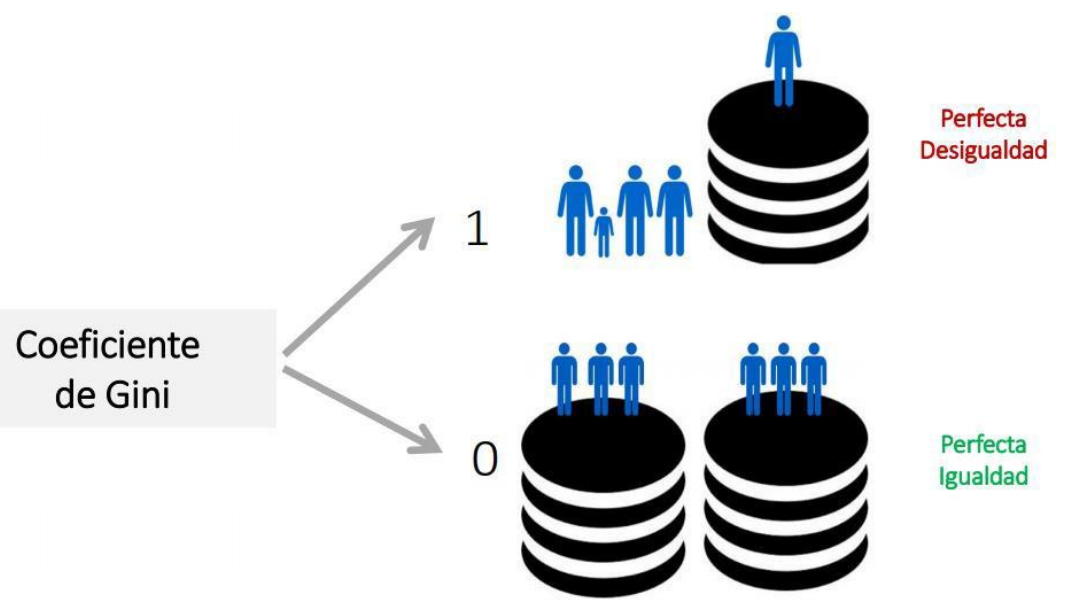

Figura 1. Representación gráfica del índice de Gini

\section{Coeficiente de Gini: origen, evolución, institucionalización.}

La desigualdad entre la población ha constituido uno de los problemas principales a nivel general que ha ocasionado pobreza entre los ciudadanos en los diferentes territorios. La pobreza es 
el resultado de una mala distribución de la riqueza que acumulan las personas, asimismo las insuficientes políticas públicas destinadas a mejorar las condiciones sociales en gran medida son consecuencia también de la aparición de la pobreza.

A nivel de país, la desigualdad, constituye uno de los mayores problemas dentro de la sociedad, situación que ha sido influenciada directamente por la inestabilidad política registrada por los diferentes gobiernos que a su debido tiempo han pasado por el poder, además de las políticas económicas que poco han sido direccionadas al interés social y sobre todo a la realidad nacional. Se puede mencionar que una de las consecuencias de esta situación recayó en la migración internacional que se produjo, en donde aproximadamente dos millones de compatriotas salieron en busca de mejores oportunidades, por otro lado, el fortalecimiento del flujo interno migratorio a las ciudades grandes que acumulan un porcentaje mayoritario de la población además de agrupar la actividad económica del país (Sarmiento Moscoso, 2017).

Bajo este contexto, según Ramírez \& Díaz (2017) establecen que “cuando se habla de 'desigualdad de la renta', simplemente se refiere a las diferencias de renta, sin tener en cuenta su deseabilidad como sistema de recompensas o su indeseabilidad como esquema que contradice cierta idea de igualdad" (p. 15)

En la antiguiedad a pesar que existieron grandes diferencias entre factores ambientales y geográficos, no era tan visible la presencia de desigualdad entre sus pobladores, sin embargo, a nivel de las tribus de aquellas épocas ya se empezaba a evidenciar la desigualdad en el poder, dado que el poder influía en gran medida en la repartición de recursos.

Posterior a la época primitiva, las nuevas formas de sociedades que se constituyeron a pesar de tener ciertas discrepancias de jerarquía y poder, a nivel de sociedades no se registraban 
principales problemas de desigualdad, sin embargo, luego de la caída del sistema feudal, aparece la competencia internacional. En donde, en Inglaterra nace la revolución industrial, en la cual dentro de las características principales que tuvo dicha revolución constan: la revolución agrícola y la revolución demográfica, donde se pasó de una economía rural basada en la agricultura y el comercio, a una economía urbana, industrial y mecanizada (Oliván, 2016).

A partir de la revolución industrial, las sociedades hegemónicas empiezan a crecer a pasos agigantados, mientras que el resto de países que no pertenecen a este tipo de sociedad lo hacen en menor proporción, es ahí que se van formando los primeros rasgos de desigualdad entre países.

Habitualmente cuando se utiliza la palabra desigualdad es para señalar la manera en que están asignados los recursos de una sociedad, relacionando a dicha asignación temas de consumo, ingreso o riqueza, en el marco de una idea subyacente de igualdad entre las personas. Bajo este contexto, la desigualdad social inmiscuye diferentes áreas que intentan mostrar el nivel de diferenciación social entre personas, lo que da información sobre su grado de pertenencia a una clase o estatus social determinado. Estas áreas cubren un arco muy amplio que incluye aspectos relacionados con el acceso a educación, trabajo, salud, defensa jurídica, libertad de expresión, derechos de propiedad, entre otros. El grado de calidad de estos aspectos es determinante para establecer el nivel de vida tanto de los individuos como de la comunidad (Tezanos, Quiñones, Gutierrez, \& Madrueño, 2013).

En este sentido, las desigualdades sociales, aparecen por las formas de acumulación que se dan en los diferentes procesos de producción de las economías, en donde quienes están al frente de la producción (dueños) acumulan en mayor porcentaje las riquezas, además, la escaza intervención 
del estado y las políticas públicas aplicadas no han sido de gran ayuda para tratar de reducir las brechas de desigualdad que se registran en las diferentes economías al día de hoy.

En la literatura económica con frecuencia este problema pasa por alto, y prácticamente no se registran estudios acerca la desigualdad económica que se acompañen de información que haga referencia a la precisión estadística del índice de Gini, o de cualquier otro indicador que dé cuenta del nivel de desigualdad entre el grupo de hogares analizados. Durante los últimos años, este tema se ha empezado a discutir con mayor frecuencia, y se muestra la necesidad de incorporar elementos que comuniquen sobre la calidad estadística de los indicadores, al tiempo que se está consciente de que el tratamiento para datos agrupados resulta más complejo que cuando se trabaja con observaciones individuales. Es decir, la mayoría de estudios que atienden esta problemática tratan de aproximar el error estándar de los coeficientes de desigualdad a partir de las propiedades asintóticas de distribuciones muéstrales, por lo que la aplicación de los resultados teóricos que se han derivado está condicionada a la disponibilidad de información para muestras suficientemente grandes en las que el supuesto de normalidad de las distribuciones se satisfaga. (Medina, 2001)

Por otro lado, el asunto de la concentración del ingreso, y sus posteriores efectos a nivel social y macroeconómica, durante los últimos años ha ido ganando espacio, es así que Sarmiento (2017) establece que la concentración de ingresos genera un obstáculo grande para el crecimiento económico. Así mismo destaca que la distribución del ingreso a nivel general, es decir de forma mundial, el $20 \%$ más rico de la población disfruta de aproximadamente el $81 \%$ del ingreso, mientras que el $20 \%$ más pobre cuenta con sólo el 1\%, Además dentro de su estudio ubican a Latinoamérica como la zona más desigual del mundo. 
Se registran también, indicadores que permiten medir las brechas de desigualdad, mismos que están divididos en indicadores positivos, que según Mateo (2015) “son aquellos que no hacen referencia ni están basados en conceptos de bienestar, es decir, representa cifras estadísticas que miden una variable determinada, lo cual es muy común escuchar de información obtenida a través del cálculo de la varianza, covarianza, desviación estándar, etc.”; e indicadores normativos que según Sen \& Foster (2016) constituyen "los que sí están fundamentados en relación al bienestar, por lo que se puede considerar que son de carácter cualitativo en el cual no solamente se basa a una variable especifica sino a un serie se sujeciones propias del sistema”.

A pesar de ser una medida positiva, uno de los principales indicadores de desigualdad positivo constituye el coeficiente de Gini, “este indicador, clasifica las medidas estadísticas para el análisis de la distribución del ingreso, sin embargo, no utiliza como parámetro de referencia el ingreso medio de la distribución -a diferencia de la desviación media, la varianza y el coeficiente de variación-, ya que su construcción se deriva a partir de la curva de Lorenz". (García, Fuentes, \& Montes, 2012)

\section{Historia y Rasgos del Coeficiente de Gini}

El coeficiente de Gini toma su nombre por el estadista, sociólogo y demógrafo italiano Corrado de Gini, quien fue la persona que propuso el coeficiente como método de medida de desigualdad en el año 1912, la metodología considera la obtención de porcentajes acumulados de totales y ser comparados, a través de cocientes, con porcentajes acumulados de observaciones, dicha información genera una curva denominada "Curva de Lorenz o Curva de Concentración” (Buccioni, 2012). 
Los estudios realizados por Gini no solo permiten analizar una variable de forma estadística, sino que permite además construir distribuciones empíricas, a partir de la curva de Lorenz que analiza dos variables (población, ingreso). El coeficiente de Gini mide la desigualdad en la renta de un país, sin embargo, este coeficiente no permite medir el factor de bienestar, es decir, la identificación de quien posee acumulada las riquezas de un territorio no ayuda a determinar el grado de bienestar del mismo de forma acertada, por lo que es considerado únicamente como un indicador estadístico que permite medir el grado de concentración de la riqueza (Romero García, 2015).

\section{Parámetros de medición del Coeficiente de Gini}

Un índice de desigualdad, constituye una medida que resume la forma cómo se distribuye una variable entre un conjunto de individuos. Para el caso específico de la desigualdad económica, la medición se encuentra directamente asociada al ingreso (o al gasto) de las personas o a su vez de las familias. Bajo estas características, este indicador, constituye una de las medidas estadísticas para realizar el análisis de la distribución del ingreso, a partir de la curva de Lorenz, misma que muestra el porcentaje acumulado del ingreso total que pertenece al p\% (porcentaje de población) más pobre de la población (INEC, 2016).

Los parámetros de medición del coeficiente de Gini considera dos criterios: el primero es el criterio de Lorenz mientras que el segundo es el criterio de Lorenz generalizado, en donde se realiza una referencia entre la renta y el individuo.

El coeficiente de Gini se mide en rangos de 0 y 1 , donde 0 es considerado como igualdad máxima, es decir equidad absoluta en la que toda la población agrupa la misma cantidad de riqueza, sin embargo pensar que un determinado territorio logre un coeficiente de Gini 0, es algo muy difícil, debido que las economías actuales en un mundo globalizado no lo permite pues el capitalismo ha 
generado que los dueños de los elementos de producción acumulen mayor cantidad de riqueza que el resto de personas. Por otro lado, cuando el coeficiente es 1 , se considera que una persona reúne toda la riqueza de un país, además cuando los valores del coeficiente se acercan a uno, se puede establecer que en el territorio se encuentran presentes problemas de desigualdad, los cuales conducen a problemas de pobreza y de difícil acceso a los servicios básicos necesarios para su supervivencia.

Por su parte, el Banco Central del Ecuador (2017) define a la curva de Lorenz como "aquella representación gráfica de la desigualdad, para representar la forma como se reparte una variable entre un conjunto de personas. En este caso particular, la medición de la desigualdad económica está asociado directamente al ingreso per cápita de los individuos” (p. 15).

Bajo estas consideraciones, cada punto de la curva puede ser leído como porcentaje acumulativo ya sea de las personas o a su vez de los hogares. La curva, en sí, inicia del origen, es decir, $(0,0)$ y culmina en el punto $(1,1)$. De esta forma, si el ingreso se encontraría distribuido de forma perfectamente equitativa, la curva de Lorenz coincidiría con la línea de 45 grados; mientras que, si se registrara perfecta desigualdad, donde, una persona concentrara el ingreso total, la curva indudablemente coincidiría con el eje de las x, es decir el eje horizontal del plano cartesiano (ver figura 2). 


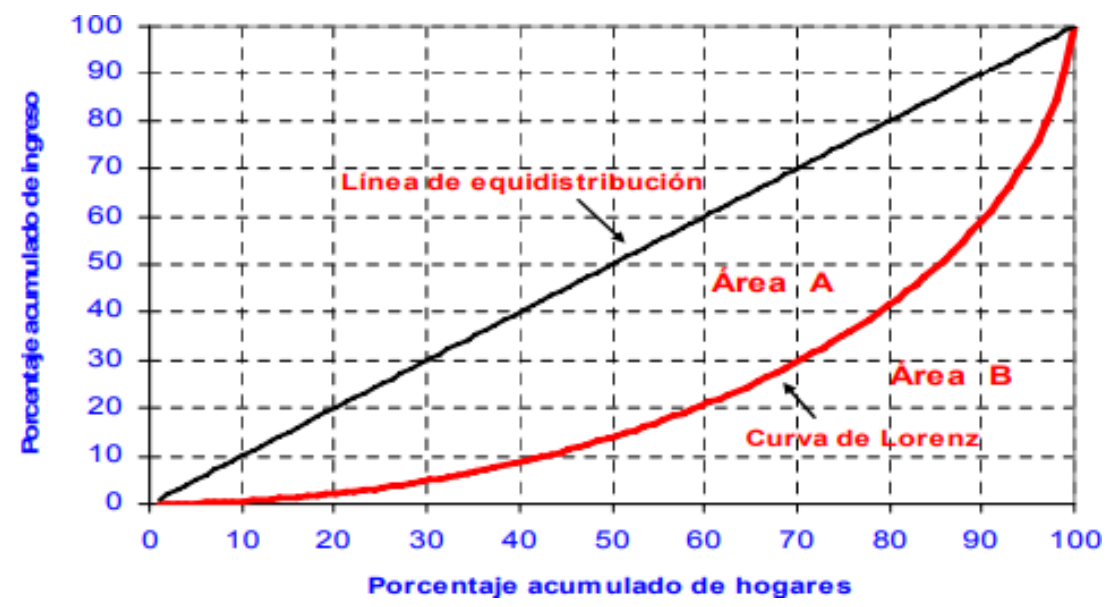

Figura 2. Representación gráfica curva de Lorenz. Tomado de: (Medina \& Galván, 2008)

Es por ello que, por medio de la curva de Lorenz se puede medir el coeficiente de Gini, "la cual muestra el porcentaje acumulado del ingreso total que pertenece al porcentaje de la población más pobre. Es el cociente entre el área comprendida de la diagonal y la curva de Lorenz (área a) sobre el área bajo la diagonal (área a+b)" (Banco Central del Ecuador, 2017) si la curva de Lorenz se acerca a la línea de perfecta igualdad, se considera una correcta distribución de los ingresos, es decir, que el coeficiente de Gini se acerca a cero, mientras que si la curva de Lorenz más se acerca al eje de las equis, menor es la distribución, por lo que existe mayor desigualdad en los ingresos de las personas, por lo tanto, el coeficiente de Gini se acerca a uno. Estructurar un diagrama para representar la distribución del ingreso resulta una opción útil para realizar el análisis de la desigualdad, dado que ayuda a identificar ciertos aspectos de la forma de la distribución que de otra forma no sería factible visualizar. (Medina, 2001)

Bajo este contexto, una de las formas para el cálculo respectivo del coeficiente de Gini, es a través de la curva de Lorenz, la cual permite graficar la distribución de los ingresos por grupos de personas, estas pueden estar representadas por quintiles o por deciles, por ejemplo, el decil 1 acumula el $50 \%$ de las riquezas, cada decil está compuesto por un grupo de la población. 


\section{Metodología del Coeficiente de Gini}

Para calcular el coeficiente de Gini, se puede considerar algunas metodologías (fórmulas), propuestas por diferentes autores:

Fórmula de Brown, constituye la más común para realizar el cálculo del coeficiente de Gini y cuya fórmula de es la siguiente:

$$
G=1-\left|\sum_{k=1}^{n-1}\left(X_{k+1}-X_{k}\right)\left(Y_{k+1}+Y_{k}\right)\right|
$$

Donde:

G: Coeficiente de Gini

Y: Proporción acumulada de la variable ingreso.

k: Quintil / Decil de Estudio

X: Proporción acumulada de la variable población.

Por otro lado, si se parte desde el análisis de la curva de Lorenz, donde el coeficiente de Gini ayuda a medir la proporción que existe entre la curva de perfecta distribución y la curva de Lorenz, se obtiene como coeficiente de cálculo la siguiente expresión:

$$
\text { Gini }=\frac{a}{a+b}
$$

Donde

$\mathrm{a}=$ Curva de Lorenz

$\mathrm{b}=$ Proporción del área de bajo de la diagonal

Bajo este contexto, cuando la curva de Lorenz esté más cerca a la curva de perfecta distribución, el coeficiente de Gini se acercará a cero, por el contrario, si más alejada esta la curva de Lorenz a la curva de perfecta distribución, el coeficiente de Gini se acercará a uno. 
Es decir, equivale a la siguiente ecuación:

$$
\left.G=1+\left(\frac{1}{n}-\left(\frac{2}{n^{2} \breve{y}}\right) \bigotimes_{1}+y_{2}+\ldots+n\right)_{n}\right) \text { donde } y_{1} \geq y_{2} \geq y_{n}
$$

Donde

$\mathrm{n}=$ Número de población

y = Ingresos por grupos de población

y̆ $=$ Ingreso medio de la población

Por otro lado, Corrado de Gini en 1912 estableció como fórmula para realizar el cálculo del coeficiente la siguiente expresión:

$$
C G=\frac{1}{2 \mu}\left[\frac{\sum_{i=1}^{n} \sum_{j=1}^{n}\left|y_{i}-y_{j}\right|}{n(n-1)}\right]=\frac{1}{2 \mu} \Delta
$$

Donde:

$\Delta=$ media aritmética de $\mathrm{n}$

$(\mathrm{n}-1)=$ diferencias absolutas de las observaciones

$2 \mu=$ Valor máximo que asume $\Delta$ cuando una persona concentra los ingresos

$\mathrm{y}=$ Ingresos observados

Por su parte, el mismo Gini en 1914 mejora el indicador para el cálculo del coeficiente de desigualdad estableciendo la siguiente formula para el cálculo respectivo

$$
C G=1-2 F(y)
$$

Donde:

$\mathrm{F}(\mathrm{y})=$ Curva de Lorenz

Por lo que, el coeficiente de Gini representa 1 menos dos veces la curva de Lorenz.

Consecutivamente, se define la derivación de la fórmula de Gini de la siguiente forma:

$$
C G=\frac{D M R}{2}=\frac{\frac{\sum_{i, j}\left(y_{i}-y_{j}\right)}{\breve{y}} \frac{1}{n^{2}}}{2}=\frac{\sum_{i, j}\left(y_{i}-y_{j}\right)}{2 n^{2} \breve{y}}
$$

Es decir, el coeficiente de Gini puede ser definido como el coeficiente de las diferencias

entre los valores de la curva de Lorenz y la línea de equidistribución. Además, se registran varias formas de derivar de manera algebraica el índice de Gini y una de ellas afirma que es exactamente 
igual a la mitad de la diferencia media relativa (DRM) la que se define como la media aritmética de las diferencias absolutas entre todos los pares de ingresos.

De acuerdo con Ferreira \& Garín (1997) con base a la información de la curva de Lorenz y estableciendo el incremento de los ingresos de las personas, se puede calcular el coeficiente de Gini mediante las frecuencias relativas acumuladas y simples, esto mediante el (pi - qi), es decir, se obtiene la siguiente fórmula de cálculo:

$$
G=\left(\frac{\sum\left(p_{i}-q_{i}\right)}{\sum\left(p_{i}\right)}\right)
$$

Por otro lado, se considera un colectivo de tamaño $\mathrm{N}$ y una variable $\mathrm{X}$ objeto de análisis con la notación siguiente:

$X_{1}<X_{2}<\ldots<X_{k}$ : $\mathrm{k}$ valores distintos que adquiere la variable estadística $\mathrm{x}$

$$
n_{1}, n_{2}, \ldots, n_{k}: \text { frecuencias absolutas asociadas }
$$

$p_{1}, p_{2}, \ldots, p_{k}$ : frecuencias relativas $\left(p_{1}=\underline{n}_{N_{i}}, \operatorname{con} N=\sum_{i=1}^{k} n_{i}\right)$. Por tanto, $\sum_{i=1}^{k} x_{i} n_{i}$ resulta ser el total (masa salarial, renta,...) que se reparte entre el colectivo

$q_{1}$ :masa relativa repartida entre los miembros de la clase $i$ - ésima; es decir,

$$
q_{i}=\frac{x_{i} n_{i}}{\sum_{j=1}^{k}\left(x_{j} n_{j}\right)}
$$

Una manera de visualizar, el grado de uniformidad del reparto es a través de la representación de la curva de Lorenz. En la cual se representan los puntos $\left(P_{i} Q_{i}\right)$, donde $P_{i}=$ $\sum_{j \leq i} p \quad$ y $Q_{i}=\sum_{j \leq i} q_{j}$

Con estos antecedentes si se quiere apuntar que la diagonal principal del cuadrado $(0,1 * 0,1)$ representaría la equidad total, es decir se evidenciaría un reparto uniforme, mientras que una mayor 
cercanía a los ejes representaría en sí una concentración mayor en el reparto generando desigualdad entre las variables calculadas.

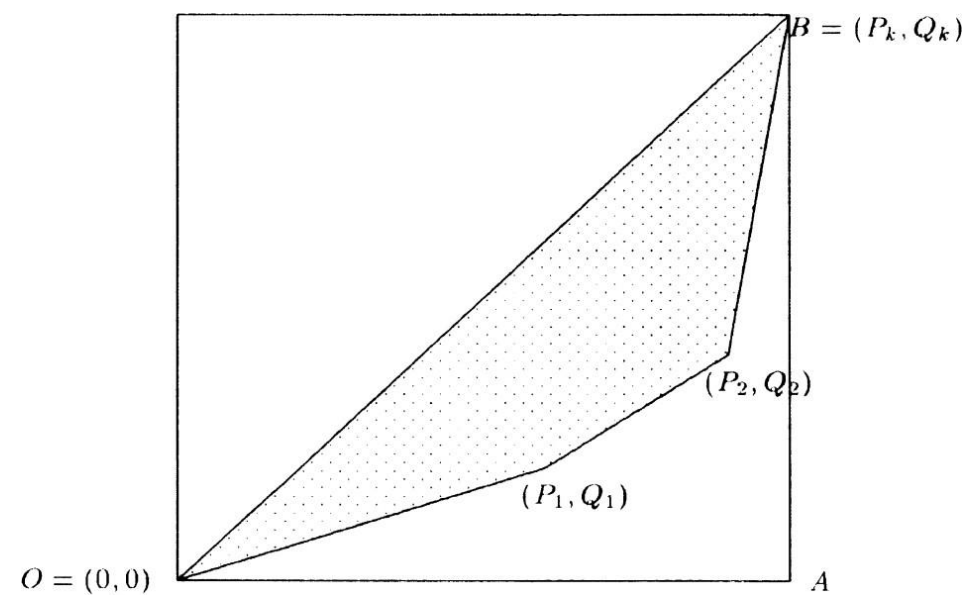

Fundamentado en la curva de Lorenz y en que la concentración se puede visualizar en términos del área que se produce entre la diagonal y ella (área rayada). La definición general del índice de Gini en la estadística descriptiva constituye la siguiente:

$$
l_{G}=\frac{\text { área rayada }}{\text { área } O A B}
$$

Donde $\mathrm{OAB}$, constituye el triángulo bajo la diagonal principal, sin embargo, diferentes autores como (Escuder, 1982; Muñiz \& Uriel, 1988, Martín, 1994, Montiel et. al., 1997), establecen la siguiente ecuación para obtener el índice de Gini:

$$
l_{G 1}=\frac{\sum_{i=1}^{k-1}\left(P_{i}-Q_{i}\right)}{\sum_{i=1}^{k-1} P_{i}}=1-\frac{\sum_{=1}^{k-1} Q_{i}}{\sum_{i=1}^{k-1} P_{i}}
$$

Por otro lado, otra de las fórmulas comúnmente utilizadas, constituye aquella propuesta por (Rodríguez \& Arenales, 1988), los cuales destacan la siguiente ecuación para realizar el cálculo respectivo: 


$$
l_{G 2}=\frac{\sum_{i<j}\left(x_{j}-x_{i}\right) n_{i} n_{j}}{N(N-1) x}
$$

A nivel del país, a diciembre de 2017, el coeficiente de Gini nacional fue de 0.459, en el área urbana fue de 0.435 mientras que en el área rural fue de 0.463. en relación a diciembre de 2016, las variables estadísticamente no son tan significativas INEC (2017). A continuación, en la tabla 1, se describen los valores del índice de Gini obtenidos durante los años 2016 y 2017.

\begin{tabular}{|c|c|c|c|c|c|}
\hline \multirow[t]{2}{*}{ Indicador } & Área & Dic 2016 & Dic 2017 & Diferencia & p-valor \\
\hline & Nacional & 0,466 & 0,459 & $-0,66$ & 0,419 \\
\hline \multirow[t]{2}{*}{ Coeficiente de Gini } & Urbano & 0,450 & 0,435 & $-1,48$ & 0,128 \\
\hline & Rural & 0,456 & 0,463 & 0,71 & 0,596 \\
\hline
\end{tabular}

El índice de Gini conforme se acerca a 1, significa que las sociedades son más desiguales y conforme su valor se aproxima a 0 , son menos desiguales, por lo general los países desarrollados poseen un índice de Gini entre 0,24 y 0,3 tal es el caso de Noruega con un valor de 0,24, mientras que los países con mayor desigualdad en el mundo como es el caso de Brasil alcanzan índices de hasta 0,59 (Gaibor, García, \& Songor, 2017). Bajo este contexto, la evolución que se puede observar de la desigualdad en el Ecuador de forma general por medio del índice de Gini poblacional tomando en consideración los ingresos totales en relación al año 2016 ha disminuido dado que paso de 0,466 en el año 2016 a 0,459 en el año 2017.

\section{Conclusiones.}

En relación al origen, evolución e institucionalización del Coeficiente de Gini, se destaca información acerca de la desigualdad y como esto fue ganando espacio poco a poco con el pasar de 
los años, desde la época primitiva donde no se registraba mayor desigualdad a excepción de las tribus quienes empezaban a evidenciar la desigualdad por el poder que mantenían hasta llegar a la revolución industrial donde se pasó de una economía rural basada en la agricultura y el comercio, a una economía urbana, industrial y mecanizada, es decir, las sociedades hegemónicas empezaban a crecer a pasos agigantados, mientras que el resto de países lo hacían en menor proporción, es ahí donde se fueron formando los primeros rasgos de desigualdad entre países.

Por otro lado, la historia y rasgos del coeficiente de Gini, empieza con su creador Corrado de Gini quien en el año 1912 propuso el coeficiente con su apellido el cual permitió calcular la desigualdad en la renta de un país, sin embargo, este coeficiente no permite medir el factor de bienestar, es decir, la identificación de quien posee acumulada las riquezas de un territorio no ayuda a determinar el grado de bienestar del mismo de forma acertada, por lo que es considerado únicamente como un indicador estadístico que permite medir el grado de concentración de la riqueza (Romero García, 2015).

Los parámetros de medición del coeficiente de Gini se centra en rangos de 0 y 1, donde el primero considera igualdad máxima, es decir equidad absoluta o correcta distribución de ingresos dado que la curva de Lorenz se acerca a la línea perfecta de igualdad, mientras que el segundo considera que una persona agrupa toda la riqueza de un país, es decir esta relación determina que un territorio presenta problemas de desigualdad los cuales pueden conllevar a problemas de pobreza y de difícil acceso a servicios básicos para la supervivencia de un grupo de personas.

Finalmente, en Ecuador se puede observar que de la desigualdad de forma general por medio del índice de Gini poblacional tomando en consideración los ingresos totales en relación al año 2016 ha disminuido dado que paso de 0,466 en el año 2016 a 0,459 en el año 2017. 


\section{Referencias Bibliográficas.}

Banco Central del Ecuador. (2017). Reporte de pobreza, ingreso y desigualdad. Quito. Retrieved from https://contenido.bce.fin.ec/documentos/Estadisticas/SectorReal/Previsiones/IndCoyuntura/Em pleo/PobrezaDic2017.pdf

Buccioni Vadulli, R. (2012). Estimación del coeficiente de concentración de Gini a partir de la curva estimada de Lorenz. Revista Chilena de Economía Y Sociedad, 5(1-2), 27-31. Retrieved from http://search.ebscohost.com/login.aspx?direct=true\&db=fua\&AN=89477637\&lang=es\&site=eh ost-live

Cortés, F., \& Rubalcava, R. M. (2016). El coeficiente Gini-Intervalo; la forma de la concentración a través de una descomposición del índice de Gini. Demografía Y Economía, XVI, 1-26.

Ferreira, E., \& Garín, M. A. (1997). Una nota sobre el cálculo del índice de Gini. Estadística Española, 39(142), 207-218.

Fuenzalida, M., Buzai, G., Morero Jiménez, A., \& García de León, A. (2015). Geografía, geotecnología y análisis espacial: tendencias, métodos y aplicaciones. (Triángulo, Ed.).

Santiago de Chile. Retrieved from http://s3.amazonaws.com/academia.edu.documents/40177316/Fuenzalida_et_al._2015_Geogra fa_Geotecnologa_y_Anlisis_Espacial.pdf?AWSAccessKeyId=AKIAIWOWYYGZ2Y53UL3A $\&$ Expires $=1488255322 \&$ Signature $=2$ UY1\%2Bcg3suaW7duHLRKVkHpw3z4\%3D\&responsecontent-disposition=inli

Gaibor, J., García, P., \& Songor, X. (2017). Remesas ¿salida de la desigualdad en países latinoamericanos? Un estudio comparativo entre Ecuador y México. Revista nuestrAmeríca, 5, $140-159$.

García, A., Fuentes, N., \& Montes, O. (2012). Desigualdad y polarización del ingreso en México: 1980-2008. Política $Y$ Cultura, 285-310. Retrieved from http://content.ebscohost.com/ContentServer.asp?T=P\&P=AN\&K=77946216\&S=R\&D=a9h\&E bscoContent=dGJyMNLr40Sep7c4xNvgOLCmr0uep7NSsqu4S7OWxWXS\&ContentCustome r=dGJyMPGnsEq3r7BRuePfgeyx 44Dt6fIA\%5Cnhttp://search.ebscohost.com/login.aspx?direct $=$ true $\& d b=a 9 h \& A N=7794621$

INEC. (2016). Indicadores de Pobreza- Junio 2016.

INEC. (2017). Reporte de Pobreza y Desigualdad, 13.

Instituto Nacional De Estadísticas Y Censos INEC. (2017). Encuesta Nacional de Empleo, Desempleo y Subempleo Indicadores de Pobreza y Desigualdad Junio 2017, 29. Retrieved from http://www.ecuadorencifras.gob.ec/documentos/web-inec/EMPLEO/2015/Junio2015/201506_EnemduPresentacionN_15anios.pdf 
Mateo, C. M. (2015). Análisis e incidencia de la desigualdad a través del índice de Gini en la ciudad de Guayaquil, período 2011-2015. Universidad de Gauayaquil.

Medina, F. (2001). Estudios estadísticos y prospectivos. (CEPAL, Ed.), Estudios estadísticos y prospectivos (Vol. 4). Santiago de Chile. Retrieved from http://www.cepal.org/publicaciones/xml/8/14038/lc2024e.pdf

Nabernegg, M. (2015). El efecto de las políticas de Preferencia Nacional en las Compras Públicas en Ecuador 2009-2011: Desconcentración y Componente Nacional. Análisis Estadístico, X, 59-93.

Oliván Cortés, R. (2016). La cuarta revolución industrial, un relato desde el materialismo cultural. URBS. Revista de Estudios Urbanos Y Ciencias Sociales, 6(2), 101-111. Retrieved from http://www2.ual.es/urbs/index.php/ urbs/article/view/olivan

Ramírez Álvarez, J., \& Díaz Sánchez, J. (2017). Fuentes de la desigualdad económica en Ecuador. Economía Y Política, XIII, 9-28. https://doi.org/10.25097/rep.n25.2017.01

Romero García, J. E. (2015). Medidas estadísticas derivadas de indicadores de desigualdad. Universidad de Sevilla.

Sarmiento Moscoso, S. (2017). Evolución de la desigualdad de ingresos en Ecuador, período 20072015. Analítica, 13(1), 31. Retrieved from http://www.ecuadorencifras.gob.ec/documentos/webinec/Revistas/Analitika/Anexos_pdf/Analit_13/2.pdf

Sen, A., \& Foster, J. (2016). La desigualdad económica. (Fondo de Cultura Económica, Ed.). México.

Tezanos Vázquez, S., Quiñones Montellano, A., Gutierrez Sobrao, D., \& Madrueño Aguilar, R. (2013). Desarrollo humano, pobreza y desigualdades. (COIBA, Ed.). Santander. Retrieved from http://www.academia.edu/2773444/Desarrollo_humano_pobreza_y_desigualdades 